\title{
Longevity Studies in the CDF II Silicon Detector
}

\author{
Satyajit Behari on behalf of the CDF Collaboration \\ The Johns Hopkins University, Baltimore, MD 21218. \\ E-mail: behari@fnal.gov
}

\begin{abstract}
The CDF Run II silicon detector is the largest operating detector of its kind in High Energy Physics, collecting $p \bar{p}$ collision data at the Fermilab Tevatron since 2001. It provides precision tracking and vertexing which played a critical role in the $B_{s}$ mixing discovery and is essential to the ongoing Higgs Boson search and many other physics analyses carried out at CDF. Due to the prolonged Tevatron Run II program the detector faces unforeseen challenges while operating well beyond its design parameters. Of particular concern is the radiation aging of the silicon sensors which are expected to acquire $\sim 10 \mathrm{fb}^{-1}$ data, far above their design integrated luminosity of $2-3 \mathrm{fb}^{-1}$. In this paper we discuss the impact of radiation damage to the sensors, their effect on the physics performance and expectations for future operations of the two inner layers, which have already inverted.
\end{abstract}

Index Terms-CDF Run II, Silicon strip detectors, radiation damage.

\section{The CDF Run II SiLICON Detectors}

$\mathbf{T}$ HE Collider Detector at Fermilab (CDF) [1] is one of the two multi-purpose particle physics detectors at the Tevatron collider of Fermilab. The Tevatron has delivered an integrated luminosity of $7 \mathrm{fb}^{-1}$ per experiment so far and is expected to operate beyond 2010, delivering data in excess of $9 \mathrm{fb}^{-1}$. The CDF Run II silicon detector provides precision tracking and vertexing information essential for most of CDF's broad physics program. It is also used in the CDF Silicon Vertex Trigger (SVT) [2], the first hardware-implemented displaced vertex trigger at a hadron collider detector.

The silicon detector is comprised of three sub-detectors with a total of $722 \mathrm{k}$ readout channels from $7 \mathrm{~m}^{2}$ of silicon sensors. A ladder is the smallest detector building block consisting of 2-4 sensors, 4-16 readout chips and read out via a High Density Interconnect (HDI). The Layer 00 (L00) [3] is installed directly on the beam pipe. L00 uses radiation hard, single-sided $p$-in- $n$ sensors from Hamamatsu at radius $1.62 \mathrm{~cm}$ and SGS Thomson at radius $1.35 \mathrm{~cm}$ fabricated with design parameters similar to those in use by the Compact Muon Solenoid (CMS) [4] silicon tracker at the LHC. It also includes two oxygenated module made by Micron. The L00 sensors can be biased up to $500 \mathrm{~V}$, limited by the power supply. SVX-II [5] is the core sub-detector and the only component used in the SVT. SVX-II has 5 layers of double-sided $p$-in$n$ sensors located at radii between $2.5-10.6 \mathrm{~cm}$. The layers 0,1 and 3 are made of Hamamatsu sensors with the $n$ strips perpendicular to the $p$ strips. The layers 2 and 4 are made of Micron sensors with a $1.2^{\circ}$ angle between the $n$ and $p$ strips. The Hamamatsu and Micron sensors can be biased up to 170 $\mathrm{V}$ and $70 \mathrm{~V}$, respectively, limited by the breakdown voltage of the integrated coupling capacitors and subtle sensor effects. ISL [6] provides an extended forward tracking coverage where
CDF wire chamber has partial coverage. ISL has one central layer at a radius of $22 \mathrm{~cm}$ and two forward layers at radii of $20 \mathrm{~cm}$ and $28 \mathrm{~cm}$. They are made of double-sided $p$-in- $n$ Hamamatsu and Micron sensors with the $n$ strips at a stereo angle of $1.2^{\circ}$ with respect to the $p$ strips.

\section{IMPORTANCE OF LONGEVITY STUdies}

The Tevatron has delivered an integrated luminosity of 7 $\mathrm{fb}^{-1}$ so far and is expected to run through 2010 , with a possible extension to 2011. This would expose the CDF silicon detectors to radiation doses proportional to the collected data in excess of $9 \mathrm{fb}^{-1}$, far beyond their design specifications of $3 \mathrm{fb}^{-1}$. In addition, aging electronics and cooling system, reducing spare pool etc. pose significant challenges for an efficient operation of the detectors [7]. Figure 10 shows the current fractions of integrated (black), good (green) and bad (red) ladders vs. run number. A ladder is considered good

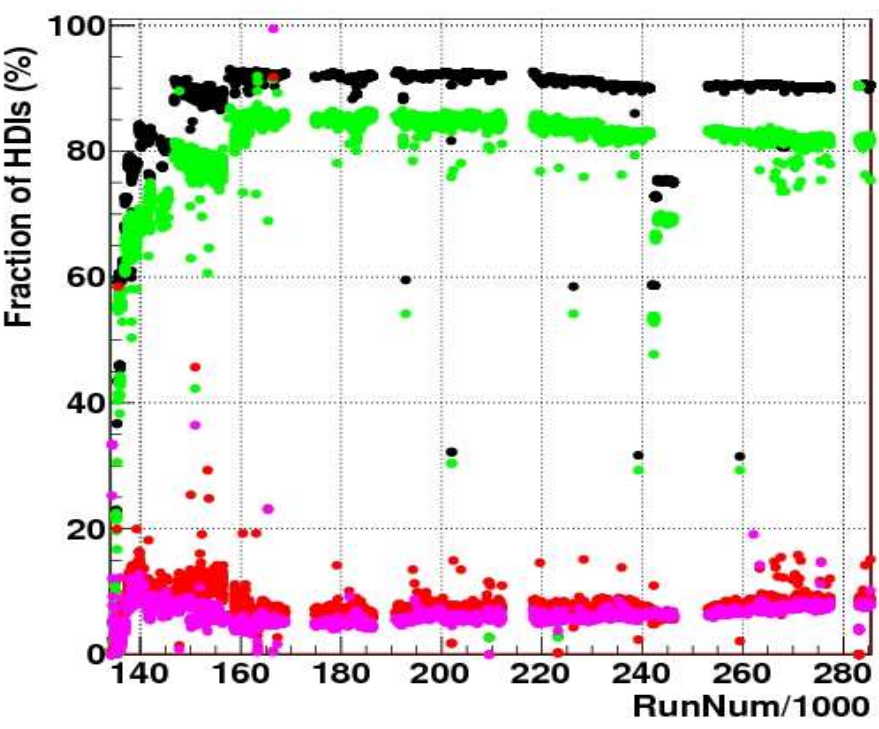

Fig. 1. The fraction of integrated (black), good (green) and bad (red) ladders vs. run number. Shown also is the average digital error rate (magenta).

if the data acquired by it suffer from $<1 \%$ digital errors and bad otherwise. Shown also is the average error rate (magenta). Over the past 8 years of operation, the good ladder fraction has reduced by $3 \%$, indicating an excellent overall performance since the commissioning.

The Tevatron Run II program was originally expected to run till 2009 and accumulate $15 \mathrm{fb}^{-1}$ of data. This would have required replacement of the inner layers of the CDF silicon 
detectors. A revised Tevatron luminosity profile projected a much lower target luminosity of $4-8 \mathrm{fb}^{-1}$ by 2009 and led to the cancellation of the CDF Run IIb silicon upgrade project [8] in September 2003. In 2004 a panel of CDF silicon experts made a set of recommendations to improve the detector longevity. They include running the detector significantly colder, implement fixes to some identified DAQ channel failure modes, safeguard against Tevatron beam incidents and regularly monitoring changes in sensor depletion voltage. These recommendations were carried out promptly. Nevertheless the radiation hardness of the sensors remains the single most important issue for future operations.

\section{EFFECTS OF RADiATiOn DAMAGE}

The CDF silicon detector is located in an intense radiation field, dominated by $p \bar{p}$ collisions as opposed to beam losses. Measurement of the ionizing radiation dose in the CDF detector hall was carried out early in Run II [9] by means of over 1000 thermal luminescent dosimeters (TLDs) spread over the entire detector volume. An extrapolation of the measured dose using an inverse power law in radius gives an estimate for the ionizing radiation dose of $300 \pm 60$ $\mathrm{kRad} / \mathrm{fb}^{-1}$ at a radius of $3 \mathrm{~cm}$ with a $20 \%$ variation over $z$. This means that L00 and Layer-0 of SVX-II have already received doses of $\sim 7 \mathrm{MRad}$ and $\sim 2.8 \mathrm{MRad}$, respectively, after $7 \mathrm{fb}^{-1}$ of Run II luminosity. The flux to luminosity factor has been measured [10] using the increase in bias currents of the sensors, resulting in $0.93 \pm 0.26\left(10^{13} 1 \mathrm{MeV} n\right.$ equiv.) $/ \mathrm{cm}^{2} / \mathrm{fb}^{-1}$ for the Layer- 0 of SVX-II. The inner sensors of L00 receive 2.5 times higher flux. The integrated fluence in L00 after $8 \mathrm{fb}^{-1}$ will be of the order of what is expected for the LHC strip trackers.

Radiation damage to the sensor bulk causes the number of effective charge carriers to change, gradually decreasing the voltage required to deplete the sensor until type inversion, after which the depletion voltage increases. The Layer- 0 of SVX-II is of most concern for this type of damage because it is located at a radius of $2.5 \mathrm{~cm}$ from the beam-line and its maximum bias voltage is limited to $170 \mathrm{~V}$, as mentioned in Section I Defects in silicon crystals due to radiation damage lead to an increase of the sensor leakage current and, as a consequence, to an increase of sensor shot noise. In addition, signal strength degrades as crystal defects cause degradation of charge collection efficiency. These two effects, in combination with increasing readout ASIC noise, cause a degradation of the signal-to-noise ratio $(S / N)$. Therefore, two major concerns for the longevity of the CDF silicon detector are the ability to fully deplete the silicon sensors and degradation of the signalto-noise ratio.

The performance of the detector is not expected to change significantly due to degradation of the readout electronics by the integrated radiation dose. Of particular concern are the readout ASIC and the Dense Optical Interface Module (DOIM) which transmit optical data to the front-end electronics crates. The noise in the readout ASIC is predicted to increase by $17 \%$ [11] and the light output from the DOIMs to drop by $10 \%$ [12] after $8 \mathrm{fb}^{-1}$. Other radiation induced

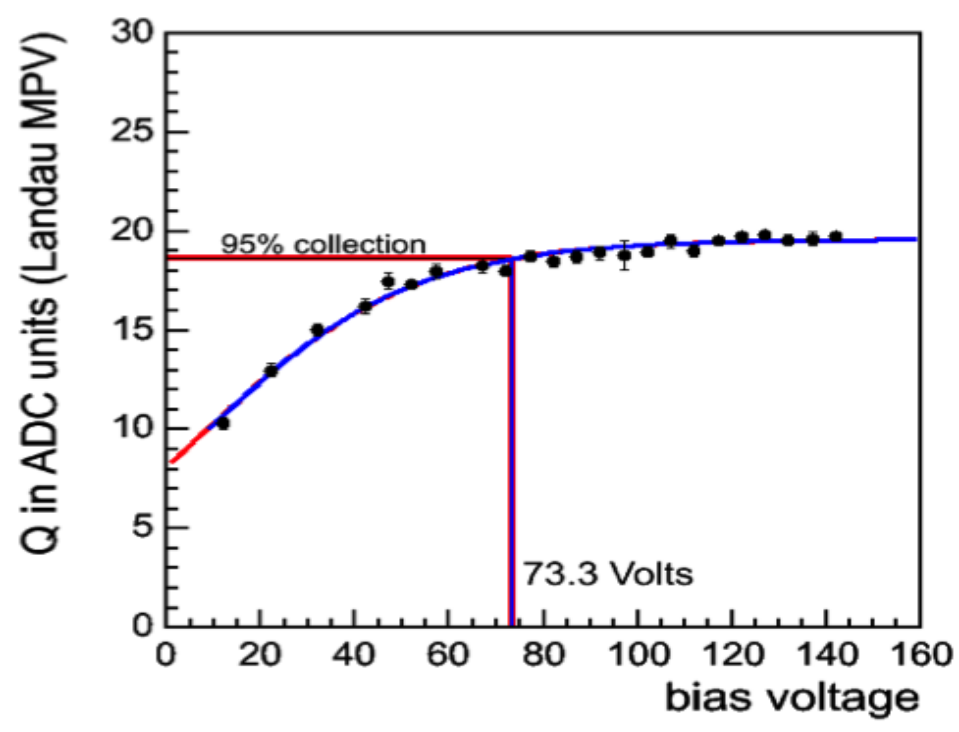

Fig. 2. Determination of the depletion voltage for a ladder. It is defined as the bias voltage at which $95 \%$ of the charge collection plateau is reached.

effects manifest themselves as increase in Single Event Upset (SEU) rates in the front-end electronics and power supply crates located in the CDF detector hall. So far there has been no alarming signs of increase in these rates.

\section{RESUlts AND Discussion}

For a given ladder the depletion voltage is measured in the following way. The charge distribution is fitted to a Landau function convoluted with a Gaussian resolution function. The resulting Most Probable Value (MPV) is plotted against a range of applied bias voltages, as shown in Figure 2. This distribution rises with bias voltage and saturates to form a plateau at higher voltages. The depletion voltage is defined as the bias voltage that collects $95 \%$ of the charge at the plateau. The time evolution of depletion voltage is studied as a function of the integrated luminosity. A $3^{\text {rd }}$ order polynomial fit is performed to extract the sensor substrate type inversion point. A linear fit is applied to data points beyond $+1 \mathrm{fb}^{-1}$ from the inversion point to obtain future predictions. Figures 3 and 4 show the evolution of the depletion voltage for L00 and SVXII Layer-0, respectively. The positive slope of the depletion voltage evolution indicates that all the CDF Run II silicon sensors have passed their type inversion. Due to our condition for the linear fits, the inversion point is roughly $1 \mathrm{fb}^{-1}$ below the starting point of the lines. It is interesting to note that the CMS-type oxygenated Micron L00 ladders (shown in red) invert much later compared to the other two, indicating their superior radiation hardness. It is clear from these plots that we will be able to fully deplete most of the L00 and SVX-II Layer-0 sensors until $10 \mathrm{fb}^{-1}$.

For a given side, $r-\phi$ or $z$, and layer of SVX-II the signal-to-noise ratio $(S / N)$ is measured in the following way. The signal, $S$, is measured as the charge collected from hits on muon tracks reconstructed in $J / \psi \rightarrow \mu^{+} \mu^{-}$events collected using CDF di-muon trigger. The extracted signal from a Landau fit is path corrected and decreases linearly 


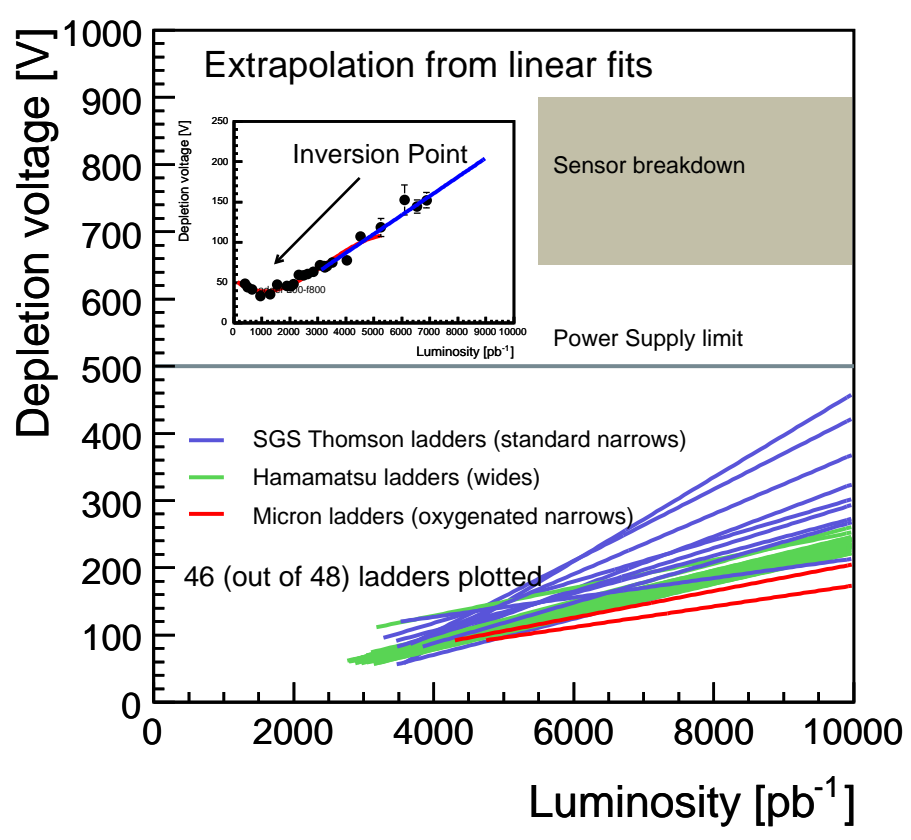

Fig. 3. Prediction of L00 sensor depletion voltage up to $10 \mathrm{fb}^{-1}$. The 3 makes of the sensors are shown in different colors. Shown also are power supply limit of $500 \mathrm{~V}$ and the sensor breakdown region.

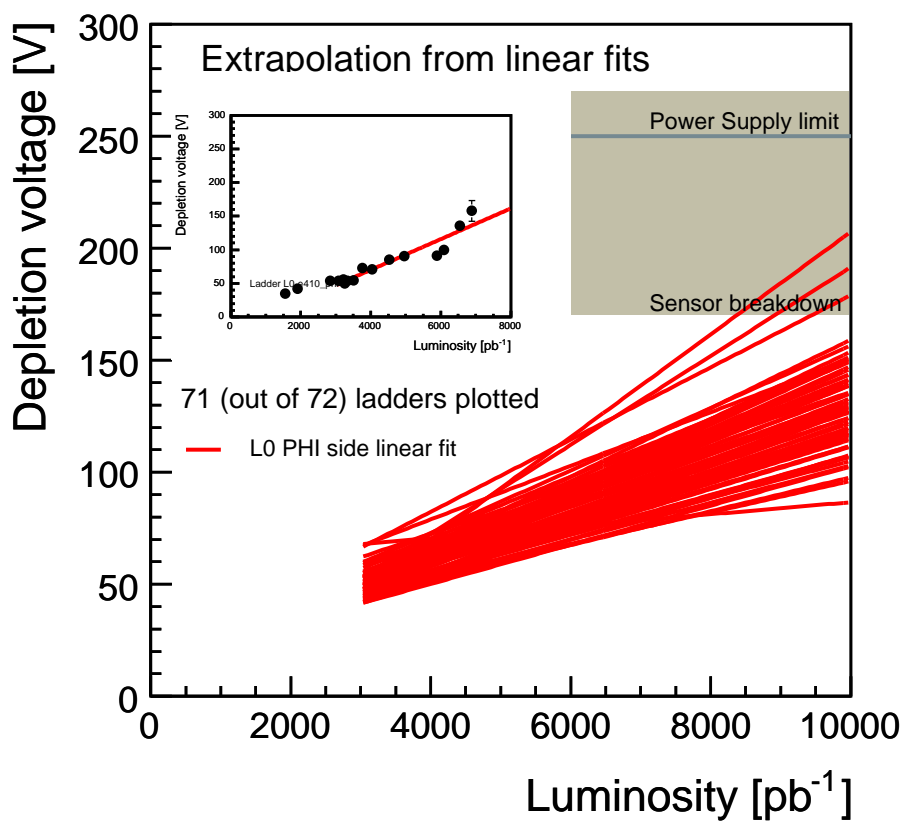

Fig. 4. Prediction of SVX-II Layer-0 sensor depletion voltage up to $10 \mathrm{fb}^{-1}$. Shown also are power supply limit of $250 \mathrm{~V}$ and the sensor breakdown region (starts from $\sim 170 \mathrm{~V}$ ).

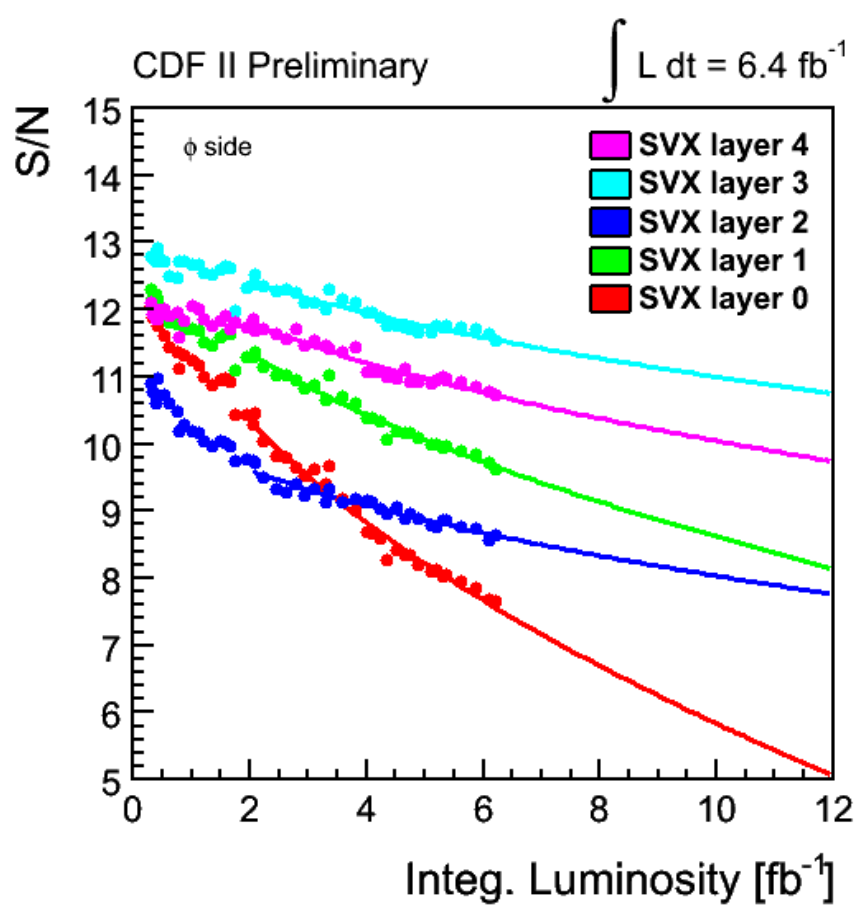

Fig. 5. Prediction of $S / N$ for the $r-\phi$ strips of SVX-II up to $12 \mathrm{fb}^{-1}$.

with integrated luminosity. The noise, $N$, averaged over charge clusters in a ladder is estimated using special calibration runs with beam. It increases as a square-root with the integrated luminosity. Figures 5 and 6 show the evolution of $S / N$ for the $r-\phi$ and $z$ strips of SVX-II, respectively, up to $12 \mathrm{fb}^{-1}$.

The plots show the predicted $S / N$ ratios above 5 up to an integrated luminosity of $10 \mathrm{fb}^{-1}$. This would correspond to a tracking performance adequate for $b$-tagging, based on CDF Run I experience, and for using SVX-II (especially Layer-0) in the SVT hardware trigger [13] for the remainder of Run II.

\section{CONCLusions}

Radiation aging studies show the CDF Run II silicon detectors are in good health after 8 years of operation. Studies of depletion voltage and $S / N$ ratio evolution have been instrumental in maintaining optimal detector performance through bias voltage upgrades. The L00 and Layer-0 of SVX-II have long progressed through type inversion and exhibit consistent post-inversion behavior. Most SVX-II Layer- 0 ladders would be fully depleted up to $10 \mathrm{fb}^{-1}$. The innermost layer, L00, is expected to compensate for any lost tracking and vertexing performance. In summary, the CDF Run II silicon detector will continue successful operation for the rest of Run II, beyond 2010. 


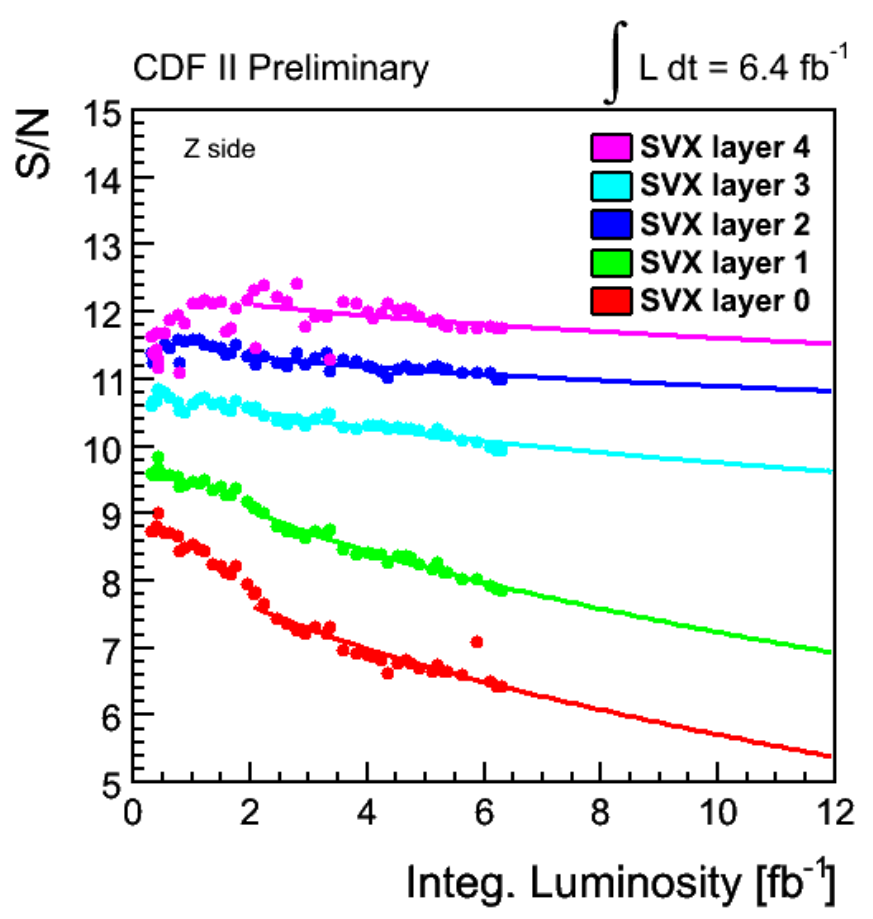

\section{REFERENCES}

[1] D. Acosta et al., "Measurement of the cross section for $t \bar{t}$ production in $p \bar{p}$ collisions using the kinematics of lepton + jets events", Phys. Rev. D72 052003, 2005.

[2] W. Ashmanskas et al., "The CDF silicon vertex trigger", Nucl. Instrum. Meth. A518 532-536, 2004.

[3] C.S. Hill et al." "Initial experience with the CDF Layer 00 silicon detector", Nucl. Instrum. Meth. A511 118, 2003.

[4] R. Adolphi et al.,"The CMS experiment at the CERN LHC", JINST 3 S08004, 2008.

[5] A. Sill et al.,"CDF Run II silicon tracking projects", Nucl. Instrum. Meth. A447 1-8, 2000.

[6] A. Affolder et al. "Intermediate silicon layers detector for the CDF experiment", Nucl. Instrum. Meth. A453 84-88, 2000.

[7] M.J. Mathis, "Operational Experience with the CDF Run II Silicon Detector", N13-165 poster presentation, IEEE-NSS-2009 conference.

[8] T. Akimoto et al., "The CDF Run IIb Silicon Detector: Design, preproduction, and performance", Nucl. Instrum. Meth. A556 459-481, 2006.

[9] R.J. Tesarek et al.,"A measurement of the radiation environment in the CDF tracking volume", Nucl. Instrum. Meth. A514 188-193, 2003.

[10] P. Dong et al., "An Analysis of Bias Currents in SVX II", CDF internal note 8219, 2006.

[11] M. Garcia-Sciveres et al." "The SVX3d integrated circuit for deadtimeless silicon strip readout", Nucl. Instrum. Meth. A435 58-64, 1999.

[12] R.S. Lu" "Status and radiation hardness of the CDF Run II silicon detector", Nucl. Instrum. Meth. A552 219-223, 2005.

[13] A. Mitra, "The CDF Run II Silicon Detector", N18-1 presentation, IEEENSS-2006 conference.

Fig. 6. Prediction of $S / N$ for the $z$ (stereo) strips of SVX-II up to $12 \mathrm{fb}^{-1}$. 